\title{
Prestigio culturale ed esigenze normative nelle tradizioni linguistiche regionali italiane Un'esperienza di ricerca
}

\section{Fiorenzo Toso}

Centro Internazionale sul Plurilinguismo

Università di Udine

\begin{abstract}
Il saggio ricostruisce le tappe del percorso storico-linguistico attraverso il quale il genovese si propone costantemente, a partire dalla seconda metà del sec. XII, come elemento costitutivo nel disegno complessivo di una specificità culturale ligure assunta a simbolo e motivazione di un quadro socio-politico marcato, nella percezione interna ed esterna, da una consapevolezza forte di alterità. Il caso specifico ha valore soprattutto se inquadrato in una visione olicentrica della cultura italiana, nella quale le differenti storie linguistiche regionali non vengano viste esclusivamente come conseguenza di una tensione centripeta uniforme, ma anche come frutto di dinamiche di volta in volta originali nel rapporto fra tradizione linguistica locale - dotata o meno di un proprio autonomo prestigio - e orizzonte idiomatico soprarregionale.
\end{abstract}

Parole chiave: genovese, storia linguistica della Liguria, letterature regionali, letteratura genovese, plurilinguismo.

\section{Abstract}

This essay reconstructs the halting historico-linguistic journey through which Genovese has constantly been deemed, from the second half of the C. XII onwards, a constitutive element in the general design of a Ligurian cultural specificity considered both symbol of and motivation for a marked socio-political framework, within an internal and external perception, of a strong consciousness of alterity. This specific case has the additional value of being framed within a polycentric vision of Italian culture, in which the differing regional linguistic histories are not perceived exclusively as the consequence of a uniform, centripetal tension, but also as the outcome of original dynamics in the relation between local linguistic tradition - provided at least with its own, autonomous prestige - and the supraregional idiomatic horizon.

Key words: Genovese, the linguistic history of Liguria, regional literature, Genovese literature, plurilingualism. 
Una rilettura della storia linguistica italiana organizzata per regioni è stata tentata di recente nel lavoro collettivo diretto da Francesco Bruni, ${ }^{1}$ che ha rappresentato indubbiamente, insieme ad altre pubblicazioni di questi ultimi anni, un progresso notevole verso una percezione policentrica della cultura linguistica del paese, sul solco di una tradizione illustre che dopo gli antesignani ottocenteschi, dal Ferrari in poi, aveva già offerto risultati significativi in alcuni aurei contributi d'insieme di Carlo Dionisotti, Mario Sansone e altri. È innegabile tuttavia che la prospettiva adottata in questo genere di studi rimane di preferenza quella di un'analisi delle modalità di «italianizzazione» delle singole regioni, come denuncia il titolo stesso dell'opera (L'italiano nelle regioni) e la ricostruzione di volta in volta proposta delle diverse modalità attraverso le quali le differenti realtà culturali regionali approdano, nel corso di processi secolari di adeguamento, a una loro collocazione all'interno del panorama linguisticoletterario nazionale.

All'attivo di questa e di altre esperienze di ricerca va senz'altro ascritta la presa d'atto che i processi di adeguamento alla prospettiva linguistica nazionale si verificarono secondo tempi e modalità profondamente diversi a seconda delle singole aree: il che potrebbe sembrare un'osservazione di per sé banale, ma che non lo è affatto se si tiene conto dei pesanti retaggi della visione storico-linguistica italiana più divulgata fino all'altro ieri. Ciò che manca in genere è piuttosto la percezione di un'autonomia delle singole "storie linguistiche» regionali, e soprattutto delle singole «storie della lingua» che si potrebbero elaborare per le diverse varietà destinate a confrontarsi con l'italiano nel corso di un processo plurisecolare di convergenza: mentre si ammette la pluralità delle esperienze dell'italiano nelle varie regioni non si pone a sufficienza l'accento, a mio parere, sulle diverse modalità dell'interrelazione che, nel corso di questo processo, si attua tra l'italiano e gli attori locali, percepiti, questi ultimi, come modalità linguistiche invariabilmente collocate sullo stesso piano nel loro rapporto con la lingua nazionale e i suoi progressi. La banalizzazione del rapporto lingua-dialetto - ed è stata più volte osservata l'insufficienza dell'opposizione terminologica, poiché se l'italiano è "uno», sotto il concetto di dialetto si pongono invece realtà estremamente diversificate, non soltanto sul piano diatopico - implica infatti un appiattimento delle condizioni di partenza e degli sviluppi dei processi storico-linguistici, mentre invece proprio i tempi e le modalità diverse di italianizzazione delle singole regioni dovrebbero suggerire l'esigenza di verificare, tra le altre concause, la capacità reattiva di dialettalità variamente collocate dal punto di vista diastratico, da quello percettivo, da quello della funzionalità come strumento di comunicazione non meno che di (auto)riconoscimento: è evidente che la storia linguistica regionale del Piemonte è diversa da quella dell'Abruzzo, ad esempio, non solo per le modalità diverse dell'interrelazione tra fattori idiomatici e realtà socio-economica: que- 
st'ultima ha variamente condizionato, prima e contemporaneamente alle modalità di assunzione dell'italiano, il prestigio del piemontese e dell'abruzzese.

Non tutte le varietà regionali italiane - a prescindere dalla maggiore o minore distanza tipologica dallo standard - si collocano quindi sullo stesso piano e si mostrano dotate di analogo prestigio; inoltre, fatto non meno significativo, non tutte le varietà dialettali italiane entrano storicamente in gioco nella «costruzione» di una identità culturale di lunga durata, tale da disegnare l'ipotesi di una appartenenza che si configuri almeno in parte come «altra» rispetto all'orizzonte nazionale: quando ciò avviene, la lettura della storia linguistica regionale propone variabili importanti, e suggerisce la possibilità di una prospettiva di lettura diversa rispetto a quella che si propone semplicemente di ricostruire i processi di affermazione dell'italiano nella regione in questione.

Alla storia linguistica della Liguria e alla storia del genovese ho dedicato da alcuni anni a questa parte una serie di contributi, sia visioni d'insieme che approfondimenti di singoli aspetti: l'esemplarità e al tempo stesso la tipicità del caso meritavano di essere messi nella giusta evidenza anche in prospettiva metodologica, per fare emergere non tanto la presunzione di un'originalità, quanto la possibilità, a partire da uno specifico esempio, di fornire chiavi di lettura valide anche per altre realtà regionali, in modo da suggerire un'interpretazione storico-linguistica spostata sulle differenti storie delle lingue locali. Il quadro d'insieme che emerge da questo tentativo è abbastanza indicativo, infatti, delle prospettive di lettura e di interpretazione che si aprono grazie al rovesciamento del punto di vista, che si ottiene riformulando i termini del confronto linguistico tra italiano e genovese (veneto, piemontese, siciliano...) invece che tra italiano e dialetto in Liguria. All'interno poi di questa prospettiva, la storia della "costruzione» di una immagine del genovese, a sua volta intimamente legata alla "costruzione» di una determinata immagine della genovesità, è l'aspetto che meglio determina le modalità del rapporto con l'italiano, attribuendo continuità e coerenza a un disegno storico che, lungi dall'essere radicalmente opposto a quello tradizionale sulle modalità dell'italianizzazione linguistica della regione, ne offre nondimeno una lettura profondamente originale.

Il sunto che offro in questa sede - col costante riferimento, per eventuali approfondimenti e ulteriori rimandi bibliografici, ai saggi e ai contributi nei quali ha trovato via via sistemazione la ricostruzione storica promossa - è un primo tentativo di sintesi organica, ed ha principalmente lo scopo di fare emergere per induzione alcune implicazioni generali sul problema "storico", non meno che attuale, del policentrismo culturale italiano, e sull'esigenza di una rinnovata attenzione nei confronti di esso.

Già la tradizione linguistica medievale genovese e ligure prefigura le condizioni di una specificità non meno affermata e coscientemente ribadita che effettivamente instaurata: essa si svolge all'insegna di un sostanziale disimpegno rispetto alle esperienze di koiné padana, ${ }^{2}$ verso le quali le convergenze si veri- 
ficheranno solo in maniera episodica e tardiva. Dante, accreditando attraverso la sua visione geolinguistica un netto distacco del volgare ligure rispetto al contesto settentrionale, sembra cogliere il rapporto che Genova instaura autonomamente verso l'area galloromanza da un lato e verso quella toscana dall'altro, all'intersezione di correnti culturali e linguistiche che, dopo l'instaurazione di un'autonoma tradizione letteraria con l'Anonimo Genovese, alla fine del Duecento, attraverso i volgarizzamenti trecenteschi inseriscono la città in un meccanismo di assunzione e ridistribuzione di testi variamente marcati, per quanto attiene alla loro fruizione, sull'asse diastratico.

La percezione di questa specificità linguistica è chiara nel poeta duecentesco per il quale «lo nostro latin volgar» $(156,15)$ è fondamentale strumento di fissazione e promozione dell'ideologia comunale elaborata da Iacopo da Varagine nel suo manuale di politica cittadina, la Chronica civitatis Ianuensis. La traduzione «in lingua nostra ${ }^{3}$ di testi provenzali di devozione non denuncia del resto un'adesione neppure formale alla tradizione occitanica, con la quale restano palesi, quando denuncia «[...] le vanitae / e le canzon chi son trovae / chi parlan de van amor / e de bexicii con error» $(144,189-192)$ le incolmabili distanze culturali già evidenziate del resto, e trasferite a livello parodistico, da Rambaut de Vaqueiras nel suo contrasto bilingue. Rafforzato nell'affermazione come lingua commerciale e diplomatica, nel corso del Trecento il volgare genovese aspira decisamente a mantenere e potenziare le prerogative di autonoma grammaticalità conseguite con l'opera del poeta duecentesco, sebbene sia sempre più chiara l'apertura culturale verso l'area centro-italiana: l'esigenza stessa di «tradurre» testi di devozione provenienti dall'area toscana è il sintomo più evidente di un mancato raccordo sul piano linguistico. Alla metà del secolo, i frammenti di una grammatica volgare e di un glossario testimoniano l'esigenza di una fissazione che faccia fronte, almeno in prospettiva didattica, al degrado di una "relativa compattezza» e all'affermarsi «di tradizioni grafiche diverse e spesso contrastanti, in una serie pressoché infinita di episodi individuali, dove i diversi ingredienti culturali possono entrare in combinazione in maniera estremamente varia». ${ }^{4} \hat{\mathrm{E}}$ l'epoca del resto in cui le prime riflessioni metalinguistiche sul volgare fanno capolino nelle rielaborazioni di palinsesti latini, francesi, catalani e toscani ad opera di autori locali: per Girolamo da Bavari, ad esempio, che scrive "per comu-

liani. Storia struttura uso, Torino: UTET, 2002, p. 196-225. Sulle origini del volgare e il periodo medievale: Fiorenzo Toso, Storia linguistica della Liguria, I. Dalle origini al 1528, Recco: Le Mani, 1995. Per la storia della letteratura in genovese: Fiorenzo Toso, La letteratura in genovese. Ottocento anni di storia, arte, cultura e lingua in Liguria, Recco: Le Mani, 1999-2001.

3. Per la traduzione di testi giullareschi provenzali a carattere devoto da parte dell'Anonimo si veda Aurelio RONCAGLIA, "De quibusdam provincialibus translatis in lingua nostra», in AA.VV., Letteratura e critica. Studi in onore di Natalino Sapegno, Roma: Bulzoni, 1985, vol. II, p. 1-36; Paola AllegretTI, «Modelli provenzali dell'Anonimo Genovese», Medioevo romanzo, n. 22, 3, 1998, p. 3-15.

4. Gianfranco FolenA, "Nota linguistica», in CASSIANO DA LANGASCO e Paolo RoTONDI, La consortìa deli foresteri a Genova, Genova: Sigla Effe, 1957, p. 101. 
na hutilitae de li homi e de le femene cossí como de quilli chi sam letera como de quilli chi no la sam", è evidente la difficoltà di trasferire in genovese il messaggio originario, in quanto "queste vertue no pò miga bem l'omo in romam sí propriamenti anomar, como l'entendimento de questa parola lo dixe in latim».5 A sua volta, l'anonimo volgarizzatore della Cronaca di Martin Polono esplicita le proprie istanze puristiche in un brano che contiene tra l'altro la prima attestazione del glottonimo genovese: le storie sono infatti tradotte «de profunda gramayga in jairo vorgà çenoeyse (chiaro volgare genovese), et no sença grandissima breyga. Et se per aventura in tuta questa opera [...] se ge trovasse arcuny vocaboli gasmureschi (stranieri), prego che alo scritor e alo translataor sea perdonao». ${ }^{6}$

Resta dimostrato come durante il Quattrocento i processi di adeguamento a una norma sopralocale, promossi da alcuni esponenti della modesta temperie umanistica locale, vengano deliberatamente ritardati e risultino addirittura osteggiati a livello istituzionale, nel tentativo di mantenere al genovese cancelleresco margini significativi di prestigio rispetto al volgare d'impronta settentrionale e allo stesso toscano. ${ }^{7}$ Il perseguimento di una via locale alla dialettalità riflessa, precocemente promosso dal più prestigioso cenacolo umanistico ligure attorno al 1430, si scontra così con le palesi esigenze di affermazione di una specificità linguistica che è da considerare un riflesso delle frustrazioni politiche di una classe dirigente divisa tra una precaria gestione diretta del potere e l'appoggio tattico a signorie straniere. ${ }^{8}$

L'esigenza di instaurare un rapporto fra identità culturale (in senso lato) e identità linguistica è tale, che nel progressivo degrado della grammaticalità locale «dai tersi nitori dell'Anonimo, da quella scripta salda e perspicua e, si direbbe, superbamente equilibrata fra tradizione e innovazione» ${ }^{9}$ fino a un livello di convergenza ampia verso il toscano, scandito dall'opera di autori come Andreolo Giustiniani Banca e Bartolomeo Falamonica Gentile, si finirà per attribuire a una lingua «italam nostram», ossia alla facies locale di un volgare italiano con tracce più o meno vistose di inflessione locale il ruolo di "materna lingua», come farà in particolare ai primi del Cinquecento un intellettuale di prestigio continentale quale Agostino Giustiniani, che scrive le

5. Girolamo da Bavari, vissuto nella prima metà del Trecento è da considerare l'autore, e non il semplice volgarizzatore, di un Tratao de li VII peccai mortali e di un Libro de la misera humanna cundiciom editi da Claudio MARCHIORI, Antichi volgarizzamenti genovesi da San Gerolamo, Genova: Tilgher, 1989, che qui si riprende da vol. I, p.175.

6. Cfr. Anna CORNAGLIOTTI, «Una storia biblica in antico genovese: preliminari per una edizione», in AA.VV., Miscellanea di studi offerti a Giuliano Gasca Queirazza, Alessandria: Edizioni dell'Orso, 1988, p. 181-216.

7. Su questo tema, cfr. in particolare Fiorenzo Toso, «Per una storia del volgare a Genova tra Quattro e Cinquecento", Verbum. Analecta neolatina, n. 5, 1, 2003, p. 167-201.

8. Sulle posizioni linguistiche dei primi umanisti genovesi cfr. Fiorenzo Toso, «Il volgare a Genova tra Umanesimo e Rinascimento: inflessione locale e modelli soprarregionali da Iacopo Bracelli a Paolo Foglietta», La parola del testo, n. 4, 1, 2000, p. 95-129.

9. Marzio PORRo, in Dialogo de Sam Gregorio composito in vorgà, Firenze: Accademia della Crusca, 1979. 
sue opere «non curandomi punto essere reputato toscano, sendo nato Genovese». 10

La posizione del Giustiniani, sostenitore dell'ibridismo linguistico di impronta quattrocentesca, si scontra ben presto con più motivate istanze di normalizzazione, che sono il riflesso locale dell'affermazione della teoria linguistica rinascimentale e di una Questione della lingua che si sviluppa a livello continentale. Le riforme istituzionali imposte da Andrea Doria nel 1528 suggeriscono esigenze nuove di rappresentazione simbolica dell'originale esperienza politico-istituzionale genovese, attraverso la ricerca di elementi in grado di motivarle e di dar loro legittimazione nel quadro politico-istituzionale europeo. ${ }^{11}$ Nasce allora una retorica destinata a condizionare nel tempo l'immagine stessa di Genova e dei Genovesi, che recupera da un lato, aggiornandolo, il concetto varaginiano della "perfezione» dell'esperienza politica locale, insistendo dall'altro sul concetto di elaborazione, a partire da condizioni ambientali, geografiche, socioeconomiche proibitive, di un "sistema» genovese, di una «morada de vida» fortemente marcata dalla propria peculiarità. È naturale allora che tali esigenze di autocelebrazione si incontrino con quelle legate alla ricerca di una norma linguistica che, ripudiando le esperienze tardo-quattrocentesche di volgare regionale, punti a una doppia depurazione del materiale idiomatico disponibile: in prospettiva soprarregionale, con la difesa del latino e l'adesione a un italiano sprovincializzato e ricondotto al modello bembiano; in prospettiva interna, attraverso la promozione di un genovese a sua volta ricondotto a una purezza che eluda la convergenza verso una lingua che è ormai sentita come nettamente «altra».

Le motivazioni ideologiche sottese alla promozione del genovese sono quindi decisamente più complesse del meccanico affermarsi di una percezione «riflessa» della dialettalità: ${ }^{12}$ esse hanno origine in un progetto complessivo di ridiscussione del ruolo politico della classe dirigente, nella ricollocazione della Repubblica all'interno del sistema spagnolo, nell'affermazione della «union»e

10. La definizione di lingua «italam nostram» «nostro peculiare volgare italico» ricorre nella traduzione di un documento ufficiale francese del 1499 ad opera di Stefano Bracelli figlio di Iacopo. Per la posizione del Giustiniani e la sua definizione di «materna lingua» cfr. Fiorenzo Toso, Il volgare a Genova..., cit., da cui è tratta anche la citazione dai Castigatissimi Annali della Repubblica di Genova (da p. 120).

11. Per un commento sulle problematiche istituzionali legate alla riforma costituzionale del 1528 cfr. in particolare Claudio Costantini, La Repubblica di Genova, Torino: UTET, 1986; per la storia linguistica di Genova e della Liguria nel Cinquecento, Fiorenzo Toso, «Un modello di plurilinguismo urbano rinascimentale. Presupposti ideologici e risvolti culturali delle polemiche linguistiche nella Genova cinquecentesca», in corso di stampa in Atti del Convegno di Studi "Le città plurilingui» (Udine 2002).

12. Per la critica alla concezione crociana di dialettalità «riflessa» e all'opinione ricorrente della sua origine nel Cinquecento, cfr. Fiorenzo Toso, «Diversi livelli di plurilinguismo. Lineamenti per un approccio comparativo al tema delle regionalità letterarie europee», in Furio Brugnolo e Vincenzo OrIOles (a cura di), Eteroglossia e plurilinguismo letterario. II, Plurilinguismo e letteratura. Atti del XXVIII Convegno interuniversitario di Bressanone (6-9 luglio 2000), Roma: Il Calamo, 2002, p. 459-490. 
della «libertæ» dei cittadini come presupposti ideologici della sovranità genovese. Le stesse polemiche antidialettali di Benedetto Varchi, per il quale il genovese è una lingua "barbara», "da tutte l'altre diversa», che "scrivere e dichiarare con lettere non si può» forniscono validi argomenti agli intellettuali locali coinvolti nella sua promozione, che rovesciano il giudizio dello scrittore toscano affermando l'eccellenza di una lingua la cui diversità è un pregio, e la cui barbarie originaria è stata superata grazie alle capacità dei suoi autori. La promozione del genovese frutta a Paolo Foglietta e ai suoi seguaci le lodi del Tasso, e la fortunata operazione editoriale delle Rime diverse in lengua zeneize scandisce, attraverso le numerose edizioni susseguitesi dal 1575 al 1612 il prestigio non soltanto locale di una poesia «alla genovese» lontanissima per scelta dai toni «medi» del dettato dialettale, come dimostra, oltre alle linee tematiche affrontate dagli autori raccolti e alla connotazione politica che vi soggiace, il polemico raffronto proposto con la poesia "alla veneziana», questa si francamente «dialettale», del Venier. ${ }^{13}$

Ma la promozione del genovese verso la fine del Cinquecento è solo in parte il risultato di una polemica aperta rispetto al toscano, lingua osteggiata più nei toni accesi di alcune poesie e lettere del Foglietta che in una realtà nella quale si prende atto dell'esigenza di un utilizzo dell'italiano per la stessa esportazione del modello politico ligure. Paolo tradurrà in genovese un'opera di ampio respiro celebrativo come la Genuensium Historia di suo fratello Oberto (1596), ma la versione a stampa destinata a circolare all'esterno sarà quella in toscano di Francesco Serdonati. Questo bilinguismo di fatto, che coinvolge la classe dirigente non meno dei ceti intellettuali, attraverso un uso «alto» del genovese nella prassi politico-istituzionale, salva quindi la funzione simbolica e rappresentativa della lingua locale e assicura al tempo stesso alle esigenze di comunicazione extraregionale una fruizione diffusa del toscano. Quest'ultimo dato non significa però la rinuncia alla ricerca di un'autonoma normatività, come testimoniano opere grammaticali e apologetiche quali i Paradossi sopra la lingua toscana e genovese di Francesco Buzenga (1596) e la stessa fissazione di un modello di lingua letteraria destinata ad accentuare la propria divergenza dall'uso parlato nel corso del Seicento.

In quell'epoca, il genovese di Giangiacomo Cavalli e di altri autori direttamente implicati nelle esigenze autocelebrative e rappresentative della classe dirigente presenta quindi marche fonetiche, morfologiche e lessicali arcaizzanti, che lo distinguono nettamente non solo dai dialetti provinciali - presso i rari autori che ne fanno uso il genovese diventa non a caso, col latino e l'italiano, sinonimo di lingua letteraria artificiosamente elaborata ${ }^{14}$

13. Sulla polemica contrapposizione tra i modelli «antidialettali» della poesia in genovese del Cinquecento e quelli «dialettali» in veneziano, cfr. Fiorenzo Toso, «Edizioni cinquecentesche della Strazzosa di Maffio Venier. Per un approccio al tema delle relazioni interdialettali in età rinascimentali», Atti dell'Istituto Veneto di Scienze, Lettere ed Arti, n. 158, 1999-2000, p. 121-152.

14. Sulla contrapposizione tra il genovese «lingua alta» a fianco del latino e dell'italiano e i dialetti locali come espressione di una poetica della naturalezza, cfr. Fiorenzo Toso, «Polemiche linguistiche nella Taggia del sec. XVII», Intemelion, n. 4, 1998, p. 91-105. 
ma anche dalla parlata popolare della capitale, nella quale si esprimono filoni pur corposi di poesia e di teatro "dialettale» che vivono quindi in una condizione doppiamente "riflessa», rispetto al genovese letterario e all'italiano. ${ }^{15}$ E alla lingua del Cavalli, lodata dal Chiabrera ed «esportata» nei circoli letterari italiani che pensa quindi Andrea Spinola quando raccomanda l'uso del genovese - in polemica con quello dell'italiano e dello spagnolo - persino nelle riunioni accademiche, ed è alla poesia di questo autore in particolare che si deve più che ad altri il recupero di importanti agganci con la tradizione medievale da un lato, e la fissazione dall'altro di un "canone» letterario su registri alti, la cui influenza sarà destinata a condizionare notevolmente, fino all'Ottocento e poi oltre, la percezione del genovese come lingua e della sua letteratura come portatrice di istanze e modalità spiccatamente antidialettali.

La frattura tra il genovese "polito» e la parlata popolare si ricomporrà parzialmente un secolo dopo, sia per il convergere di alcuni esiti fonetici marcati fino ad allora in senso diastratico, sia, soprattutto, per l'affermarsi di un disegno politico che intorno a Stefano De Franchi e ad altri autori «illuminati» ridisegna e aggiorna la funzione simbolica dell'idioma. L'interesse per il genovese, cresciuto ai tempi dell'orgogliosa resistenza al bombardamento francese del 1684 aveva già prodotto, nel clima preromantico di generalizzato interesse per le culture e le lingue «popolari» la riedizione dell'opera maggiore del Cavalli, la formalizzazione di un sistema ortografico aggiornato all'evoluzione fonetica del genovese e l'insorgere delle prime preoccupazioni lessicografiche, quando la sollevazione popolare del 1746-1747 contro gli occupanti austro-piemontesi ripropose con prepotenza l'esigenza di una ridiscussione delle modalità di delega del potere da parte del popolo all'oligarchia, e di un recupero di elementi simbolici in grado di cementare intorno a un programma di riforme gestite dall'alto il consenso delle classi popolari, della borghesia e dell'aristocrazia, nel segno di una rinnovata unità nazionale di tutti «ri boin cittæn amanti dra patria, dra libertæ e dra so lengua naturale». ${ }^{16}$ Il richiamo palesemente ideologico alla lingua come fattore aggregante trova riflesso nella letteratura del periodo, con un susseguirsi di poemi, poesie, canzoni e «lezendie» di contenuto patriottico, dal quale non saranno esenti neppure la traduzione non parodica della Gerusalemme liberata (1755) e la rifondazione del teatro genovese a opera del De Franchi stesso con le sue fortunate riduzioni da Molière.

15. Sui due livelli del genovese scritto secentesco (varietà alta e varietà bassa) si veda la «Nota linguistica» in Francesco Maria MARINI, Il fazzoletto, tragicommedia inedita del sec. XVII, a cura di Fiorenzo Toso e Roberto Trovato, Bologna: Commissione per i testi di lingua, 1997. Sul teatro in genovese del sec. XVII, Fiorenzo Toso, «L'utilizzo ideologico del plurilinguismo teatrale nella Genova barocca», in Vincenzo OrIOLES (a cura di), Documenti letterari del plurilinguismo, Roma: Il Calamo, 2000, p. 67-84.

16. Sulle esigenze di normalizzazione e sul rilancio del genovese come «lengua dra naçion» cfr. Fiorenzo TOSO, «Lessicografia genovese del sec. XVIII», Bollettino dell'Atlante Linguistico Italiano, III Serie, n. 22, 1998, p. 93-119. 
Sulla precettistica del 1745 e sul manifesto della nuova letteratura pubblicato nel 1772 da De Franchi come prefazione alla sua raccolta di poesie si basa quindi il programma settecentesco di défense et illustration del genovese, articolato tra l'altro nella promozione degli usi pubblici, nell'elaborazione di una letteratura sempre più aperta alla fruizione popolare, nella replica pacata alle obiezioni dei letterati toscaneggianti, nel riconoscimento della variazione interna come elemento di arricchimento della lingua. Un programma così efficace da essere esportato persino in Piemonte, regione nella quale solo allora andava irrobustendosi un modesto filone di letteratura vernacola, ${ }^{17}$ e da costituire la base per l'utilizzo ideologico del genovese — in un'ottica politica per molti aspetti contrapposta a quella defranchiana - durante il periodo dell'effimera Repubblica Ligure Democratica, nata all'ombra della Francia rivoluzionaria nel 1797 e destinata ad esserne assorbita nel $1805 .^{18}$

La successiva annessione al Regno di Sardegna — di fatto quindi alla monarchia sabauda piemontese — nel 1851, decisa d'imperio dal Congresso di Vienna, ridimensionerà brutalmente il ruolo del genovese come lingua di prestigio. Il Piemonte aveva adottato a livello ufficiale l'italiano già nel 1563, e una prassi radicata, che escludeva di fatto l'uso alto delle varietà locali negli stati sabaudi (con parziali eccezioni in Sardegna) fu così estesa anche alla Liguria. All'italiano percepito come «lingua dei Piemontesi», visti come occupanti illegittimi e come espressione di un regime decisamente più retrivo e illiberale di quello della vecchia Repubblica, si associava peraltro la consapevolezza - mentre andava avviandosi il processo risorgimentale italiano, nel quale tanti repubblicani genovesi avranno un ruolo determinante — che la definitiva adesione alla cultura e alla lingua italiana rappresentava un imprescindibile momento di convergenza nei confronti del più vasto orizzonte peninsulare e, attraverso di esso, europeo. Si verifica quindi, da un lato, un potenziamento del ruolo simbolico del genovese scritto - ormai adeguato, dopo i rivolgimenti del 1797, alle modalità dell'espressione popolare - dall'altro la ricerca di un'adesione alla cultura italiana che eluda però il raccordo con l'ambiente subalpino, considerato più arretrato e dal quale gli intellettuali genovesi si sentivano per lo più divisi da acute divergenze di ordine ideologico.

Nella prima metà del secolo, mentre la «riscoperta» delle rime duecentesche si configura, in un'epoca di generale rivalutazione (e persino di mistificazione) a livello continentale delle tradizioni medievali, come una conferma

17. Sulla dipendenza delle prime precettistiche piemontesi dal modello ideologico defranchiano cfr. Fiorenzo Toso, «Letteratura piemontese e letteratura ligure tra Sette e Ottocento. Convergenze ideologiche e tematiche, interrelazioni e suggestioni reciproche (da De Franchi a Pipino, da Calvo a Piaggio)», in Gianrenzo P. Clivio, Dario Pasero e Censin Pich (a cura di), XIV e XV Rëscontr antërnassional dë studi an sla lenga e la literatura piemontèisa (Quinsnè, 10-11 magg 1997 e 9-10 magg 1998), Ivreja: La Slòira, 2002, p. 59-80.

18. Sulla storia linguistica della Liguria in età moderna, e in particolare sull'Ottocento, cfr. Fiorenzo Toso, «Per una storia dell'identità linguistica ligure in età moderna», in Fiorenzo TOSO e William PIASTRA (a cura di), Bibliografia dialettale ligure. Aggiornamento 19791993, Genova: A Compagna, 1994, p. 1-44. 
dell'eccellenza dell'antico Comune anche in un campo come quello letterario, nel quale i Genovesi avevano sempre goduto di scarso credito, si moltiplicano così le riedizioni dei classici, le raccolte lessicali, le precettistiche ortografiche e grammaticali: i Genovesi, dopo il fallimento del tentativo secessionista del 1849 e le aperture di Cavour, aspirano così ad entrare nella futura Italia unita come portatori di un "primato" culturale e politico che passa anche attraverso la nobilitazione della loro lingua: se nessuno pretende più, ormai, di contrapporla funzionalmente all'italiano, le prove della sua eccellenza - e del suo valore simbolico identitario — si ricercano ad esempio (attraverso un'estremizzazione e distorsione dei metodi recentemente impostisi della linguistica scientifica) nella presunzione di un'antichità remotissima, che farebbe del genovese addirittura l'antenato dello stesso latino; ${ }^{19}$ se il Nigra, ministro sabaudo prima che glottologo, suggerirà all'Ascoli un'interpretazione oggi meritevole di revisione sul rapporto tra genovese e dialetti settentrionali, alla ricerca di una motivazione storico-culturale alla ritrovata unità d'intenti tra Liguri e Piemontesi, le posizioni del Diez e di altri linguisti, che vedono nel tipo ligure una varietà di transizione tra le varietà italiane settentrionali e quelle centro-meridionali forniranno nuova linfa all'immagine di una "diversità» del genovese, ${ }^{20}$ che non tarderà a essere proposta anche a livello letterario: la ventata rinascenziale che interessa nel corso dell'Ottocento le culture regionali e minoritarie europee avrà epigoni anche in Liguria — unica regione italiana interessata al fenomeno - soprattutto con la pubblicazione, nel 1870 , del poema epico $A$ Colombiade di Luigi Michele Pedevilla, le cui assonanze tematiche e ideologiche con l'Atlàntida del Verdaguer (1877) si inseriscono nel clima di un interscambio culturale ligure-catalano destinato a protrarsi nei primi anni del Novecento. $^{21}$

Di fatto però l'esperienza pedevilliana, così come quella dei periodici in genovese che si pubblicheranno a partire dal 1868 giunge in ritardo rispetto alle scelte linguistiche di una borghesia imprenditoriale che, ormai in stretta compromissione politica con la monarchia, si colloca ai vertici, con le sue compagnie commerciali e con le industrie di stato, della gestione economica del neonato stato unitario. L'italianizzazione delle classi alte (dalla quale restano esclusi peraltro gran parte dell'aristocrazia e il clero arroccato sulla difesa di valori che si ritengono minacciati dal nuovo stato liberale) sollecita quella dei ceti inferiori e può appoggiarsi, malgrado il ruolo comunicativo forte di un

19. Sulle opinioni locali relative al genovese nel corso dell'Ottocento cfr. Fiorenzo TosO, La dialettologia prescientifica in Liguria. Antologia di testi, Genova: A Compagna, 2002.

20. Cfr. Graziadio Isaia AsCOLI, «Del posto che spetta al ligure nel sistema dei dialetti italiani», Archivio Glottologico Italiano, n. 2, 1876, p. 111-160. Si tratta del primo studio nel quale il ligure viene collocato tra le varietà "galloitaliche» e di conseguenza settentrionali, in polemica con le posizioni del Diez e di altri glottologi dell'epoca.

21. Sul significato dell'opera di Pedevilla come episodio di contatto tra la cultura regionale ligure e i movimenti rinascenziali europei, cfr. Fiorenzo Toso, «Diversi livelli...», cit., p. 483490; per le relazioni culturali catalano-liguri tra Otto e Novecento, Fiorenzo Toso, «La Grammatica catalana di Gaetano Frisoni», Estudis romànics, n. 25, 2003, p. 317-325. 
idioma ancora d'uso corrente come lingua commerciale, ad esempio, in vari porti del Mediterraneo e dell'America meridionale, su agenzie efficaci quali la scuola, la burocrazia, la coscrizione obbligatoria. Questo processo di adeguamento linguistico ha come conseguenza, tra l'altro, il sorgere di una vera e propria letteratura «dialettale» che segna con Niccolò Bacigalupo in particolare il confluire della tradizione locale nelle modalità vernacolari di espressione scritta variamente affermatesi in altre regioni italiane come conseguenza dell'ulteriore appiattimento verso il basso di tradizioni linguistiche e culturali molto diversificate tra loro. La reazione a questo dato di fatto, appoggiandosi alla produzione scientifica, a opere di larga divulgazione, a nuove sistemazioni lessicali, si manifesta soprattutto nella figura e nell'opera di Angelico Federico Gazzo, autore di studi grammaticali e ortografici, di una traduzione integrale della Divina Commedia (1909) e di altre versioni rimaste manoscritte dai classici europei, volte a dimostrare come il genovese sia una «lingua romanza o neolatina come e quanto le altre, svoltasi secondo la propria indole e vivente di vita propria». 22

Al tentativo del Gazzo si riallacciano idealmente la ripresa, con Edoardo Firpo tra gli altri, di una lirica in genovese condotta su registri «alti», ma di fatto orientata, ormai, su un filone di letteratura «neodialettale» comune anche ad altre tradizioni linguistiche regionali italiane, e la nascita di un modesto associazionismo culturale che, tollerato dal fascismo (che vide tra l'altro nel genovese uno strumento per la legittimazione delle rivendicazioni irredentistiche sul Nizzardo e sulla Corsica, ove sopravvivono isole linguistiche liguri), ha continuato sino ad oggi a divulgare una immagine sostanzialmente arretrata ed edulcorata della realtà regionale, nutrita del culto di nostalgie dialettali che si riflettono in una modesta tradizione teatrale incentrata sulla «maschera» di una genovesità improbabile e retrospettiva portata al successo dall'attore Gilberto Govi.

Il rinnovato interesse scientifico - privo purtroppo di un sostanziale punto di riferimento nell'ateneo genovese, dove perdura una forte ostilità nei confronti delle problematiche linguistiche regionali - e la crescita qualitativa e quantitativa della produzione artistica in genovese (con episodi di ampia circolazione, come la nuova canzone nata dal successo delle esperienze musicali di Fabrizio De Andrè), la crescita dell'attenzione e della "curiosità» dell'opinione pubblica nei confronti del genovese e delle varietà dialettali liguri, hanno portato a diverse iniziative recenti di promozione e recupero anche se la legislazione regionale in merito alla valorizzazione e rivitalizzazione del patrimonio linguistico si presenta ancora con gravi lacune rispetto ad altri contesti italiani ed europei.

22. Sulla figura e l'opera del Gazzo, cfr. Fiorenzo Toso, «Versioni genovesi della Divina Commedia. Interpretazioni letterarie e sociolinguistiche», $A$ Compagna, N.s., 13, n. 1, 1991, p. 9-11 e 2, p. 6-8. Sulla letteratura in genovese del Novecento, oltre al vol. III di La letteratura in genovese... rimando all'antologia Emigranti do rïe. Poeti in genovese del Novecento a cura di Fiorenzo Toso, numero speciale della Rivista in forma di parole, serie IV, 19, n. 2, 1999. 